\title{
Hypoxic Wharton's Jelly Stem Cell Conditioned Medium Induces Immunogenic Cell Death in Lymphoma Cells
}

\author{
Hao Daniel Lin $(\mathbb{D}$, Chui-Yee Fong $\mathbb{D}$, Arijit Biswas $\mathbb{D}$, and Ariff Bongso $\mathbb{1}$ \\ Department of Obstetrics and Gynaecology, Yong Loo Lin School of Medicine, National University Health System, \\ National University of Singapore, Singapore 119228 \\ Correspondence should be addressed to Ariff Bongso; obgbongs@nus.edu.sg
}

Received 3 December 2019; Revised 3 March 2020; Accepted 16 March 2020; Published 20 April 2020

Academic Editor: Stefan Arnhold

Copyright ( 2020 Hao Daniel Lin et al. This is an open access article distributed under the Creative Commons Attribution License, which permits unrestricted use, distribution, and reproduction in any medium, provided the original work is properly cited.

\begin{abstract}
Mesenchymal stem cells from Wharton's jelly of the human umbilical cord (hWJSCs), and the conditioned medium (hWJSC-CM) prepared from them, were shown to be tumoricidal on many cancers. However, these tumoricidal effects were observed in hWJSCs grown under normoxic conditions of $21 \%$ oxygen in the laboratory. Since oxygen concentrations in the stem cell niche or physiological microenvironment are hypoxic and help to maintain stemness properties, the objective of this work was to evaluate whether there were differences in the tumoricidal properties of hWJSC-CM grown in $21 \% \mathrm{O}_{2}$ (normoxic) or $5 \% \mathrm{O}_{2}$ (hypoxic) environments. The results showed that hWJSCs grown under normoxic or hypoxic conditions showed no distinct morphological differences in culture and remained positive in trilineage differentiation into adipocytes, osteocytes, and chondrocytes. Hypoxic hWJSCs expressed the mesenchymal stem cell surface markers CD105, CD90, CD73, CD146, and CD108 similar to normoxic hWJSCs but were negative for the hematopoietic markers CD14, CD19, CD34, CD45, CD117, and HLA-DR. Hypoxic hWJSC-CM produced a significantly greater reduction in cell viability and a significantly greater increase in apoptosis, oxidative stress, and lipid peroxidation in human lymphoma cells compared to normoxic hWJSC-CM. Hypoxic hWJSC-CM also produced significantly greater expression of immunogenic cell death (ICD) hallmarks such as surface-bound calreticulin, HSP70, HSP90, and high mobility group binding 1 proteins and significantly decreased expression of the defense molecules CD47 and PD-L1. This study showed that the tumoricidal effect of hypoxic hWJSC-CM was superior to normoxic hWJSC-CM and should be the preferred choice of preparing hWJSC-CM for the induction of ICD on lymphoma cells.
\end{abstract}

\section{Introduction}

Primitive populations of mesenchymal stems cells have been derived from the gelatinous connective tissue matrix (Wharton's jelly) of the human umbilical cord (hWJSCs) $[1,2]$. These hWJSCs originate from the aorta-gonadmesonephros and through their movement finally come to reside in Wharton's jelly during early human development [3]. They can be harvested in large numbers, can proliferate rapidly, and have been widely used in the clinic to treat a variety of diseases as they do not form tumors and have high tolerance in transplantation settings $[4,5]$.

These hWJSCs possess tumoricidal properties. We and others have reported that hWJSCs and hWJSC-CM attenuated or abolished various carcinomas of the breast, bone, bile ducts, and bladder [6-15]. It was also reported that stem cells from the rat umbilical cord matrix induced abolishment of tumors of the mammary gland in the rat with no resulting metastases when injected intratumorally [8]. Unengineered hWJSCs homed into and reduced the tumor burden in human breast carcinomas xenografted in the rat when injected intravenously [6]. hWJSCs also stopped the proliferation of breast cancer cells by secreting dickkopf and suppressing the Wnt pathway in xenograft mice [11]. Some research groups have shown that hWJSC-CM or microvesicles derived from hWJSCs inhibited phosphoinositide 3-kinase, Akt, and Wnt/B-catenin signalling in bile duct or urinary tract cancer cells, respectively, to stop their growth $[15,16]$.

hWJSCs were also shown to release many molecules like IL-6, IL-8, and MCP-1 [17] that are involved in producing DAMPs on cancer cells and modulate the immune response in xenograft animal models of cancer [11]. hWJSC-CM have 
been shown to induce immunogenic cell death in lymphoma cells [18]. The lymphoma cells treated with hWJSC-CM undergo immunogenic cell death and exhibited "find-me/eatme"-danger-associated molecular pattern (DAMP) signals such as the surface bound calreticulin (ecto-CRT), ecto-Hsp70 and ecto-Hsp90, adenosine thiophosphate, and HMGB1 and the downregulation of PD-L1 and CD47 [18-20].

All the above studies used mesenchymal stromal or stem cells grown under normoxic conditions. However, it has been shown that the oxygen tension in the umbilical cord in which hWJSCs reside is between $1.8 \%$ and $8 \%$ (rarely exceeding 5\%) $[21,22]$. Oxygen levels in the stem cell niches or physiological microenvironment of the umbilical cord play a vital role in maintaining their stemness properties. Therefore, culturing of MSCs under normoxic conditions does not mimic the physiological microenvironment, thus promoting replicative stress, genetic instability, senescence, and cell death. More crucially, hypoxic preconditioning of MSCs prior to administration showed increased therapeutic potential in treating cardiac ischemia, critical limb ischemia, traumatic brain injury, and liver regeneration [23]. MSCs grown under hypoxic conditions secreted more tropomyosins and other proteins like endoplasmin precursor, annexin A1, transgelin, and nucleoside diphosphate kinase $\mathrm{B}$ that facilitated cardiac repair and prevented the induction of ventricular arrhythmias after myocardial infarction in the rat model [24]. Likewise, adipose-derived stem cells (ASCs) grown under hypoxic conditions secreted more anti-inflammatory and regenerative mediators like IL-6, tumor necrosis factor a, hepatocyte growth factor, and vascular endothelial growth factor that promote liver regeneration and function as compared to normoxic ASCs [25].

Bone marrow MSCs (BM-MSCs) expanded under hypoxic conditions upregulated chemokine receptors like CXCR4 and CX3CR1 and were able to enhance migration to the tumor [26]. The same study also indicated that hypoxic MSCs are more superior as carriers that deliver anticancer drugs to target tumors than normoxic MSCs. Likewise, another group also reported that adult human adipose MSCs (ASCs) cultured under hypoxic conditions had greater motility and greater homing ability to glioblastomas both in vitro and in vivo as compared to normoxic-cultured hAd-MSCs [27]. Both papers suggested that MSCs cultured close to their hypoxic physiological oxygen tension levels had higher survival, better cancer tropism, and greater anticancer effects when transplanted into in vivo animal models. Given this background, we sought to comparatively evaluate the tumoricidal effects of hWJSC-CM grown under hypoxic and normoxic conditions on human lymphoma cells.

\section{Materials and Methods}

\subsection{Cell Culture}

2.1.1. Human Wharton's Jelly Stem Cells. Human Wharton's jelly stem cell (hWJSC) lines were obtained from human umbilical cords. Written informed patient consent by the patients themselves and approval from the Singapore Ministry of Health Institutional Domain Specific Review
Board (DSRB) were obtained for this study. hWJSCs were cultured in hWJSC medium comprising of $80 \%$ DMEM, $20 \%$ fetal bovine serum (FBS) (GE Healthcare Life Sciences, Utah, USA), $1 \%$ nonessential amino acids, $2 \mathrm{mM}$ L-glutamine, $0.1 \mathrm{mM} \beta$-mercaptoethanol, $1 \%$ insulin-transferrinselenium, antibiotic/antimycotic mixture (Thermo Scientific, Rochester, NY), and $16 \mathrm{ng} / \mathrm{ml}$ basic fibroblast growth factor (Millipore Bioscience, Temecula, CA). The hWJSCs were cultured in $5 \% \mathrm{CO}_{2}$ for normoxic $\left(21 \% \mathrm{O}_{2}\right)$ and hypoxic $\left(10 \% \mathrm{O}_{2}\right.$ or $5 \% \mathrm{O}_{2}$ level) conditions using a hypoxic incubator (Heracell 240i, Thermo Scientific).

2.1.2. Human Lymphoma Cells. The use of commercial human lymphoma cell lines (Ramos, CRL-1596) (American Type Culture Collection, Rockville, MD, USA) for this study was approved by the National University of Singapore Institutional Review Board (NUS-IRB). The lymphoma cell lines were cultured in lymphoma medium consisting of RPMI (Thermo Scientific) supplemented with 10\% heat inactivated FBS (HI-FBS) (GE Healthcare Life Sciences) and 1\% antibiotic/antimycotic mixture (Thermo Scientific).

2.1.3. Human Skin Fibroblasts (CCD112sk). The use of commercial human skin fibroblasts (CCD112sk, CRL-2429) (ATCC) for this study was approved by the National University of Singapore Institutional Review Board (NUS-IRB). The skin fibroblasts were cultured in DMEM-high glucose (Thermo Scientific) supplemented with 10\% FBS (GE Healthcare Life Sciences), $2 \mathrm{mM} \mathrm{L-}$ glutamine (Thermo Scientific), and 1\% antibiotic/antimycotic mixture (Thermo Scientific).

\subsection{Characterization of Normoxic and Hypoxic hWJSCs}

2.2.1. Plastic Adherence of Cells. hWJSCs were grown under normoxic $\left(21 \% \mathrm{O}_{2}\right)$ and two hypoxic conditions $(10 \%$ or $\left.5 \% \mathrm{O}_{2}\right)$. The cells were monitored and imaged with a phase contrast microscope (Nikon Instruments, Tokyo, Japan).

2.2.2. Cell Surface Markers. The cells grown under normoxic $\left(21 \% \mathrm{O}_{2}\right)$ and hypoxic $\left(10 \%\right.$ or $\left.5 \% \mathrm{O}_{2}\right)$ conditions were first collected using trypsin (TrypLE ${ }^{\mathrm{TM}}$ Express, Thermo Scientific) for $3-5 \mathrm{mins}$ at $37^{\circ} \mathrm{C}$ in a $5 \% \mathrm{CO}_{2}$ in air, washed in phosphate-buffered saline (PBS), and blocked with $10 \%$ normal goat serum (NGS) (Thermo Scientific) for 10 mins to prevent nonspecific binding. The cells were then incubated with mouse monoclonal primary antibodies for a series of CD markers-CD14, CD19, CD24, CD29, CD34, CD40, CD44, CD45, CD49d, CD73, CD90, CD105, CD117, CD140b, CD146, CD271, HLA-DR, and CD108-PE (1:100) (BioLegend, San Diego, CA) - for 30 mins. This was followed by incubation with Alexa Fluor ${ }^{\circledR} 488(1: 500)$ goat anti-mouse secondary antibody (Thermo Scientific) for $30 \mathrm{mins}$. The cells were finally washed in PBS, resuspended in 10\% NGS, filtered using a $40 \mu \mathrm{m}$ nylon strainer (BD) to remove any cell clumps, and analyzed using a $\mathrm{CyAn}{ }^{\mathrm{TM}}$ ADP Analyzer (Beckman Coulter, Fullerton, CA).

2.2.3. Multipotent Differentiation of Normoxic and Hypoxic hWJSCs. Cells were seeded $\left(10 \times 10^{4}\right.$ cells/dish) into 6-well 
tissue culture plates and incubated at $37^{\circ} \mathrm{C}$ in a $5 \% \mathrm{CO}_{2}$ atmosphere for $24 \mathrm{hr}$ to allow for cell attachment under normoxic and hypoxic conditions. For adipogenic differentiation, the medium was changed to adipogenic induction medium containing DMEM (Thermo Scientific) supplemented with $10 \%$ FBS, $1 \%$ penicillin/streptomycin, $0.01 \mathrm{mg} / \mathrm{ml}$ insulin (Thermo Scientific), $1 \mu \mathrm{M}$ dexamethasone (Sigma), $0.5 \mathrm{mM}$ 3-isobutyl-1-methyl-xanthine (IBMX) (Sigma), and $0.2 \mathrm{mM}$ indomethacine (Sigma). The cells were cultured for 21 days, with fresh medium changes twice a week. The cells were then fixed with $4 \%$ paraformaldehyde for 10 mins, rinsed with PBS, and postfixed with $60 \%$ isopropanol for 5 mins. The cells were stained with Oil Red $\mathrm{O}$ for 5 mins, rinsed with distilled water, and counterstained with hematoxylin (Sigma).

For osteogenic differentiation, the medium was changed to osteogenic induction medium containing DMEM medium (Thermo Scientific) supplemented with 5\% FBS, $0.17 \mathrm{mM}$ L-ascorbic-acid (Sigma, St. Louis, MO), $100 \mathrm{nM}$ dexamethasone, $1 \%$ penicillin/streptomycin, and $10 \mathrm{mM}$ $\beta$-glycerophosphate (Sigma). The cells were cultured for 21 days with fresh changes of medium twice a week. Osteogenic mineralization was then evaluated by Von Kossa staining. Briefly, the cells were rinsed with PBS and fixed in $4 \%$ paraformaldehyde solution (Sigma) for 10 mins at room temperature. They were then washed with distilled water and stained in $1 \%$ silver nitrate solution (Sigma) under UV light for 60 mins. The cells were then counterstained with $1 \%$ Nuclear Fast Red (Sigma) for 5 mins.

For chondrogenic differentiation, the medium was changed to chondrogenic induction medium containing DMEM medium (Thermo Scientific) supplemented with $1 \%$ penicillin/streptomycin, 1\% insulin-transferrin-selenium (ITS), $0.17 \mathrm{mM}$ L-ascorbic-acid, $100 \mathrm{nM}$ dexamethasone, $1 \mathrm{mM}$ sodium pyruvate, $0.35 \mathrm{mM}$ proline, and $10 \mathrm{ng} / \mathrm{ml}$ transforming growth factor beta-3 (TGF $\beta-3$ ) (Sigma). The cells were cultured for 21 days with fresh changes of medium twice weekly. The cells were fixed in $4 \%$ paraformaldehyde for 30 mins and then stained with $0.5 \%$ Alcian blue (Sigma) for 30 mins at room temperature, rinsed with tap water, and then counterstained with $0.1 \%$ Nuclear Fast Red (Sigma) for 5 mins. The stained cells were subsequently visualized and imaged using bright field optics (Nikon Instrument).

2.3. Preparation of Wharton's Jelly Stem Cell Conditioned Medium ( $h W J S C-C M$ ). hWJSC-CM was prepared according to our previously described reports [9, 10]. Briefly, hWJSCs at early passages (4P-6P) were first cultured in hWJSC medium to $70 \%$ confluency in normoxic and hypoxic conditions. The medium was replaced with a serumfree basal RPMI medium (Biowest) supplemented with $1 \%$ L-glutamine and antibiotic-antimycotic mixture (Thermo Scientific). After $48 \mathrm{hr}$, the hWJSC-CM was collected, centrifuged, and filtered using $0.22 \mu \mathrm{M}$ filter (Millipore) and stored at $-80^{\circ} \mathrm{C}$ till further usage.

2.4. Exposure of Normoxic and Hypoxic hWJSC-CM on Lymphoma Cells. Lymphoma and other cell lines (fibroblast and hWJSC) were initially grown using the culture conditions recommended in published protocols to ensure that proper growth of the cells took place. Later, before the actual experiments were started to evaluate the effects of hWJSC-CM or fibroblast-CM, all cell lines (lymphoma and fibroblast) were grown in media supplemented with heat-inactivated fetal serum (HI-FBS). Thus, culture conditions were standardized. HI-FBS was used in all experiments for the experimental and control cell lines in all subsequent assays.

Ramos lymphoma cells $\left(1 \times 10^{5}\right)$ were exposed to normoxic and hypoxic hWJSC-CM supplemented with $10 \%$ HI-FBS (Thermo Scientific) for $48 \mathrm{hr}$ at $37^{\circ} \mathrm{C}$ in a $5 \% \mathrm{CO}_{2}$. The cells were then subjected to a series of evaluation parameters as described in our previously published article [18]. The cells will be tested for cell viability (MTT assay), annexin $\mathrm{V} /$ propidium iodide (PI) analysis, mitochondrial membrane potential, mitochondrial superoxide, lipid peroxidation, apoptosis (caspases 3, 8, and 9), CD47, PD-L1 surface marker expression, and immunogenic cell death DAMP (ecto-CRT, ecto-Hsp70, ecto-Hsp90, ATP, and HMGB1 secretions).

2.5. MTS Assay. For cell viability analysis, an MTS assay was performed using the Promega CellTiter 96 AQueous One Solution Cell Proliferation Assay kit (Promega Corporation, Wisconsin, USA) based on the manufacturer's instructions. Briefly, $50 \mu \mathrm{l}$ of MTS reagent was added to the respective Ramos lymphoma cell medium $(500 \mu \mathrm{l})$ and incubated for about $4 \mathrm{hr}$ at $37^{\circ} \mathrm{C}$ in a $5 \% \mathrm{CO}_{2}$ in air atmosphere. Absorbance reading at $490 \mathrm{~nm}$ using a spectrophotometer ELISA reader was taken (mQuant; BioTek, Winooski, VT, USA).

2.6. Annexin V/Propidium Iodide Assay. The samples from each experimental group were collected, washed with PBS, centrifuged, and then resuspended in $500 \mu \mathrm{l}$ of $1 \mathrm{x}$ annexin $\mathrm{V}$ binding buffer (Thermo Scientific). The cells were then stained with $1 \mu \mathrm{l}$ of annexin V-FITC (Thermo Scientific) and $0.5 \mu \mathrm{l}$ of propidium iodide (PI, Thermo Scientific) at room temperature for 15 mins. The samples were then filtered with a $40 \mu \mathrm{m}$ nylon strainer and analyzed using a $\mathrm{CyAn}^{\mathrm{TM}} \mathrm{ADP}$ analyzer (Beckman Coulter).

2.7. Functional Caspase 3/7, Caspase 8, and Caspase 9 Activity Analysis. The treated and control cells were collected and analyzed for caspase 3, 8, and 9 activities using the CellEvent $^{\mathrm{TM}}$ Caspase-3/7 Green Detection Reagent, Vybrant ${ }^{\circledR}$ FAM Caspase- 8 Assay kits (Thermo Scientific), and Caspase 9 (active) FITC Staining kit (Abcam, Cambridge, UK). Briefly, for caspase 3, the cells were incubated with $500 \mu \mathrm{l}$ of culture medium containing $0.125 \mu \mathrm{l}$ of the $2.0 \mathrm{mM}$ CellEvent $^{\mathrm{TM}}$ Caspase-3/7 Green Detection Reagent at $37^{\circ} \mathrm{C}$ for 30 mins. For caspase 8 , the cells were resuspended with $300 \mu \mathrm{l}$ of culture medium containing $10 \mu \mathrm{l}$ of $30 \mathrm{x}$ FLICA at $37^{\circ} \mathrm{C}$ for 60 mins. For caspase 9 , the cells were incubated with $300 \mu \mathrm{l}$ of culture medium containing $1 \mu \mathrm{l}$ of $30 \mathrm{x}$ FITCLEHD-FMK at $37^{\circ} \mathrm{C}$ for 60 mins. The stained cells were washed, filtered with a $40 \mu \mathrm{m}$ strainer, and analyzed using a CyAn ${ }^{\mathrm{TM}}$ ADP analyzer (Beckman Coulter).

2.8. Mitochondrial Membrane Potential Assay $(\Delta \psi)$. The mitochondrial membrane potential $(\Delta \psi)$ of the treated and control cells was collected and analyzed using the 
MitoTracker ${ }^{\circledR}$ Red CMXRos kit (Thermo Scientific). Briefly, the cells were incubated with $500 \mu \mathrm{l}$ of culture medium containing $1 \mu \mathrm{l}$ of $1 \mathrm{mM}$ MitoTracker ${ }^{\circledR}$ Red CMXRos stock solution (Thermo Scientific) at $37^{\circ} \mathrm{C}$ for 30 mins. The cells were filtered with a $40 \mu \mathrm{m}$ strainer and analyzed using a CyAn ${ }^{\mathrm{TM}}$ ADP analyzer (Beckman Coulter).

2.9. Mitochondrial Superoxide Assay. The treated and control cells were collected and analyzed for mitochondrial superoxide using the MitoSOX ${ }^{\mathrm{TM}}$ Red mitochondrial superoxide indicator kit (Invitrogen). Briefly, the cells were incubated with $100 \mu \mathrm{l}$ of culture medium containing $0.1 \mu \mathrm{l}$ of $5 \mathrm{mM}$ Mito$\mathrm{SOX}^{\mathrm{TM}}$ working solution at $37^{\circ} \mathrm{C}$ for 30 mins. The cells were then washed, filtered with a $40 \mu \mathrm{m}$ strainer, and analyzed using the $\mathrm{CyAn}^{\mathrm{TM}} \mathrm{ADP}$ analyzer (Beckman Coulter).

2.10. Lipid Peroxidation Analysis. The treated and control cells were collected and stained using the Image-iT ${ }^{\circledR}$ Lipid Peroxidation kit based on the manufacturer's instructions (Invitrogen). Briefly, the cells were stained with $10 \mathrm{mM}$ Image-iT ${ }^{\circledast}$ Lipid Peroxidation Sensor (Component A) for 30 mins at $37^{\circ} \mathrm{C}$. The cells were then washed, filtered with a $40 \mu \mathrm{m}$ strainer, and analyzed using a BD LSRFortessa ${ }^{\mathrm{TM}}$ analyzer (BD Bioscience, Heidelberg, Germany) with excitatio$\mathrm{n} / \mathrm{emission}$ filters of $581 / 591 \mathrm{~nm}$ and $488 / 510 \mathrm{~nm}$.

\subsection{Danger-Associated Molecular Pattern (DAMP) Analysis}

2.11.1. Danger-Associated Molecular Patterns (DAMPs): CRT, Hsp90, and Hsp70. The treated and control cells were collected and blocked with $10 \%$ NGS on ice for 10 mins. The cells were then incubated with primary anti-human CRT, Hsp90, and Hsp70 antibodies (1:500) (Abcam) for 30 mins on ice followed by secondary goat anti-mouse IgG $(\mathrm{H}+\mathrm{L})$ Alexa Fluor 488 antibodies $(1: 500)$ (Thermo Scientific) for 30 mins on ice in the dark. The cells were washed with PBS, resuspended in cold 10\% NGS, filtered with a $40 \mu \mathrm{m}$ strainer, and analyzed using a $\mathrm{CyAn}{ }^{\mathrm{TM}} \mathrm{ADP}$ analyzer (Beckman Coulter, Fullerton, CA, USA).

2.11.2. Extracellular ATP Concentration. The culture media from the treated and control cells were collected and stored at $-80^{\circ} \mathrm{C}$. The extracellular ATP concentration was determined using an ATP assay kit (Abcam) based on the manufacturer's instructions. Briefly, $50 \mu \mathrm{l}$ of supernatant from the treatment and control arms was mixed with $50 \mu \mathrm{l}$ of ATP reaction mix containing $45.8 \mu \mathrm{l}$ ATP Assay Buffer, $0.2 \mu \mathrm{l}$ ATP Probe, $2 \mu \mathrm{l}$ ATP Converter, and $2 \mu \mathrm{l}$ Developer Mix. The mixtures were incubated at room temperature for 30 mins in the dark. Absorbance at $570 \mathrm{~nm}$ was measured using a spectrophotometer microplate reader (mQuant; BioTek, Winooski, VT, USA).

2.11.3. Extracellular HMGB1 Concentration. The culture media in which the treated and control cells were grown were collected and stored at $-80^{\circ} \mathrm{C}$. The extracellular HMGB1 concentration was determined using an ELISA assay kit (Uscn Life Science Inc., Wuhan, China) based on the manufacturer's instructions. Briefly, $100 \mu \mathrm{l}$ of supernatant from the treatment and control arms, diluted standards, and blanks was added into the precoated 96-well strip plate for $2 \mathrm{hr}$ at $37^{\circ} \mathrm{C}$. After removal of the liquid, $100 \mu \mathrm{l}$ of Detection Reagent A was added into each well and incubated for $1 \mathrm{hr}$ at $37^{\circ} \mathrm{C}$. The wells were then washed with $350 \mu \mathrm{l}$ of $1 \mathrm{x}$ Wash Solution 3 times and blotted dry on absorbent paper. $100 \mu \mathrm{l}$ of Detection Reagent $\mathrm{B}$ was then added and incubated for $30 \mathrm{mins}$ at $37^{\circ} \mathrm{C}$. The wells were then washed with $350 \mu \mathrm{l}$ of $1 \mathrm{x}$ Wash Solution 5 times and blotted dry on absorbent paper. $90 \mu \mathrm{l}$ of Substrate Solution was then added and incubated for 20 mins at $37^{\circ} \mathrm{C}$ in the dark. Lastly, $50 \mu \mathrm{l}$ of Stop Solution was added into each well, and HMBG1 levels were measured at $450 \mathrm{~nm}$ using a spectrophotometer microplate reader (mQuant; BioTek, Winooski, VT, USA).

2.11.4. CD Marker Analysis. The treated and control cells were collected, washed once with PBS, centrifuged, and then blocked with $10 \%$ NGS on ice for 10 mins. The cells were then incubated with primary anti-human $\mathrm{CD} 47$ antibody $(1: 500)$ (BioLegend, San Diego, CA) and PD-L1 (CD274) (1:500) (BioLegend) on ice for 30 mins followed by secondary goat anti-mouse IgG $(\mathrm{H}+\mathrm{L})$ Alexa Fluor 488 antibody (1:500) (Invitrogen) for 30 mins on ice in the dark. The cells were washed with PBS, resuspended in cold $10 \%$ NGS, filtered with a $40 \mu \mathrm{m}$ strainer, and analyzed with a BD LSRFortessa $^{\mathrm{TM}}$ analyzer (BD Bioscience, Heidelberg, Germany).

2.12. Statistical Analysis. For the experiment on the surface markers of normoxic and hypoxic hWJSCs, the results were presented as the mean \pm SEM (\%) and one-way ANOVA with Bonferroni's multiple comparison post hoc analysis (SPSS Statistics v 17.0 software package) (SPSS Inc., IL) between 3 groups was used to calculate any statistically significant differences in the surface markers between normoxic and hypoxic hWJSCs. For all the other experiments, the results were expressed as the mean \pm SEM and statistical significance between the control and hWJSC-CM collected under hypoxic and normoxic groups for lymphoma cells was calculated using one-way ANOVA with Bonferroni's multiple comparison post hoc analysis (SPSS Statistics) between 4 groups. The $p$ value of $<0.05$ was considered as statistically significant.

\section{Results}

3.1. hWJSC Surface Marker Analysis. Cell surface marker analysis for MSC CD markers using flow cytometry showed that hypoxic and normoxic hWJSCs were positive for CD105, CD90, CD73, CD146, and CD108 and did not express CD34, CD45, CD14, CD19, CD117, and HLA-DR markers (Table 1). The percentage of CD surface markers between normoxic and hypoxic hWJSCs was not significantly different.

The expression of the cell surface marker (mean fluorescence index) value showed that there were significant higher expressions of CD105, CD90, CD73, CD146, and CD108 markers in hypoxic hWJSCs $\left(10 \% \mathrm{O}_{2}\right.$ and $\left.5 \% \mathrm{O}_{2}\right)$ as compared to normoxic hWJSCs. Hypoxic hWJSCs $\left(5 \% \mathrm{O}_{2}\right)$ showed the highest expression level of CD105, CD90, CD73, CD146, and CD108 markers as compared to 
TABLE 1: Flow cytometric analysis of hWJSC cell surface markers cultured in $21 \%$ oxygen, $10 \%$ oxygen, and $5 \%$ oxygen.

\begin{tabular}{|c|c|c|c|c|}
\hline Surface markers & $21 \%$ oxygen & $10 \%$ oxygen & $5 \%$ oxygen & $p$ value (one-way ANOVA) \\
\hline \multicolumn{5}{|c|}{ MSC markers (percentage (\%)) } \\
\hline $\mathrm{CD} 73$ & $98.66 \pm 0.08$ & $98.75 \pm 0.10$ & $96.34 \pm 0.29$ & n.s. \\
\hline CD90 & $98.59 \pm 0.04$ & $98.44 \pm 0.12$ & $94.83 \pm 0.14$ & n.s. \\
\hline CD105 & $95.76 \pm 0.06$ & $97.93 \pm 0.06$ & $95.43 \pm 0.15$ & n.s. \\
\hline CD108 & $96.46 \pm 0.02$ & $96.90 \pm 0.01$ & $93.20 \pm 0.01$ & n.s. \\
\hline CD146 & $94.86 \pm 0.07$ & $96.42 \pm 0.15$ & $95.57 \pm 0.10$ & n.s. \\
\hline \multicolumn{5}{|c|}{ HSC markers (percentage (\%)) } \\
\hline CD14 & $3.02 \pm 0.05$ & $2.33 \pm 0.09$ & $4.26 \pm 0.01$ & n.s. \\
\hline CD19 & $2.01 \pm 0.02$ & $2.15 \pm 0.10$ & $3.55 \pm 0.12$ & n.s. \\
\hline CD34 & $1.91 \pm 0.01$ & $1.92 \pm 0.06$ & $4.39 \pm 0.04$ & n.s. \\
\hline $\mathrm{CD} 45$ & $2.35 \pm 0.14$ & $2.32 \pm 0.15$ & $3.34 \pm 0.06$ & n.s. \\
\hline CD117 & $1.81 \pm 0.07$ & $2.39 \pm 0.08$ & $2.74 \pm 0.06$ & n.s. \\
\hline
\end{tabular}

Results are presented as the mean \pm SEM (\%). n.s.: not significant.

TABLE 2: Flow cytometric analysis of hWJSC cell surface marker expression level (MFI) cultured in 21\% oxygen, $10 \%$ oxygen, and $5 \%$ oxygen.

\begin{tabular}{|c|c|c|c|c|}
\hline Surface markers & $21 \%$ oxygen & $10 \%$ oxygen & $5 \%$ oxygen & $p$ value (one-way ANOVA) \\
\hline \multicolumn{5}{|c|}{ MSC marker expression (MFI) } \\
\hline CD73 & $90.33 \pm 0.16$ & $105.46 \pm 1.22$ & $162.36 \pm 0.49$ & $p<0.05(21 \%$ vs. $10 \%$ vs. $5 \%)$ \\
\hline CD90 & $103.23 \pm 1.32$ & $186.90 \pm 1.16$ & $313.82 \pm 1.57$ & $p<0.05(21 \%$ vs. $10 \%$ vs. $5 \%)$ \\
\hline CD105 & $17.32 \pm 0.46$ & $35.35 \pm 0.05$ & $58.59 \pm 0.48$ & $p<0.05(21 \%$ vs. $10 \%$ vs. $5 \%)$ \\
\hline CD108 & $81.29 \pm 0.24$ & $74.35 \pm 0.57$ & $188.28 \pm 1.18$ & $p<0.05(21 \%$ vs. $10 \%$ vs. $5 \%)$ \\
\hline CD146 & $44.86 \pm 5.02 E-15$ & $63.09 \pm 0.11$ & $130.16 \pm 0.19$ & $p<0.05(21 \%$ vs. $10 \%$ vs. $5 \%)$ \\
\hline \multicolumn{5}{|c|}{ HSC marker expression (MFI) } \\
\hline CD14 & $4.26 \pm 1.014$ & $4.66 \pm 0.25$ & $4.64 \pm 0.12$ & n.s. \\
\hline CD19 & $3.14 \pm 0.27$ & $3.46 \pm 0.01$ & $4.29 \pm 0.17$ & n.s. \\
\hline CD34 & $3.91 \pm 0.46$ & $3.46 \pm 0.01$ & $8.42 \pm 0.27$ & $p<0.05$ (21\% vs. $5 \%)$ \\
\hline CD45 & $2.72 \pm 0.26$ & $4.49 \pm 0.57$ & $3.57 \pm 0.21$ & n.s. \\
\hline CD117 & $3.21 \pm 0.02$ & $4.32 \pm 0.13$ & $3.84 \pm 0.06$ & $p<0.05$ (21\% vs. $10 \%)$ \\
\hline
\end{tabular}

Results are represented as the mean \pm SEM (\%). n.s.: not significant.

normoxic hWJSCs $\left(21 \% \mathrm{O}_{2}\right)$ and hypoxic hWJSCs $\left(10 \% \mathrm{O}_{2}\right)$ (Table 2). There was a significantly higher expression of CD34 marker in hypoxic hWJSCs $\left(5 \% \mathrm{O}_{2}\right)$ compared to normoxic hWJSCs $\left(21 \% \mathrm{O}_{2}\right)$ and hypoxic hWJSCs $\left(10 \% \mathrm{O}_{2}\right)$. There was a significantly higher expression of CD117 in hypoxic hWJSCs $\left(10 \% \mathrm{O}_{2}\right)$ compared to normoxic hWJSCs $(21 \%$ $\mathrm{O}_{2}$ ) and hypoxic hWJSCs $\left(5 \% \mathrm{O}_{2}\right)$ (Table 2).

3.2. Viabilities of Lymphoma Cells when Treated with Normoxic or Hypoxic hWJSC-CM. Lymphoma cells exposed to hypoxic and normoxic hWJSC-CM over $48 \mathrm{~h}$ showed significant reductions in cell viabilities compared to controls. Hypoxic hWJSC-CM $\left(5 \% \mathrm{O}_{2}\right)$ induced the greatest reduction in cell viability, and the cell viability of hypoxic hWJSC-CM $\left(5 \% \mathrm{O}_{2}\right)$ was significantly lower than normoxic hWJSC-CM $\left(21 \% \mathrm{O}_{2}\right)$ and hypoxic hWJSC-CM $\left(10 \% \mathrm{O}_{2}\right)$ (Figure $\left.1(\mathrm{a})\right)$. The fold changes in cell viabilities as compared to controls were hWJSC-CM $\left(21 \% \mathrm{O}_{2}\right): 0.88 \pm 0.02$, hWJSC-CM $(10 \%$ $\left.\mathrm{O}_{2}\right): 0.84 \pm 0.05$, and hWJSC-CM $\left(\begin{array}{lll}5 \% & \mathrm{O}_{2}\end{array}\right): 0.71 \pm 0.03$.
There were no significant differences in cell viabilities for normal skin fibroblast cells when exposed to normoxic or hypoxic hWJSC-CM over $48 \mathrm{~h}$ (Figure 1(b)). The fold changes in cell viabilities as compared to controls were hWJSC-CM $\left(21 \% \mathrm{O}_{2}\right): 1.00 \pm 0.02$, hWJSC-CM $\left(10 \% \mathrm{O}_{2}\right)$ : $1.00 \pm 0.03$, and hWJSC-CM $\quad\left(\begin{array}{lll}5 \% & \left.\mathrm{O}_{2}\right): & 1.00 \pm 0.03\end{array}\right.$ (Figure 1(b)). Lymphoma cells exposed to hypoxic and normoxic fibroblast-CM over $48 \mathrm{~h}$ showed significant increases in cell viabilities compared to the control. The fold changes in cell viabilities as compared to controls were fibroblastCM $\left(21 \% \mathrm{O}_{2}\right): 1.22 \pm 0.007$ and fibroblast-CM $\left(5 \% \mathrm{O}_{2}\right)$ : $1.24 \pm 0.001$ (Figure 1(c)).

3.3. Increased Apoptosis with Normoxic or Hypoxic hWJSC$C M$. The annexin V/PI assay of lymphoma cells treated with both normoxic and hypoxic hWJSC-CM showed significant increases in the percentages of early $(\mathrm{AV}+\mathrm{PI}-)$ and late $(\mathrm{AV}$ $+\mathrm{PI}+$ cells) apoptotic cells over controls. The hypoxic hWJSC-CM $\left(\begin{array}{ll}5 \% & \mathrm{O}_{2}\end{array}\right)$ produced significantly higher 


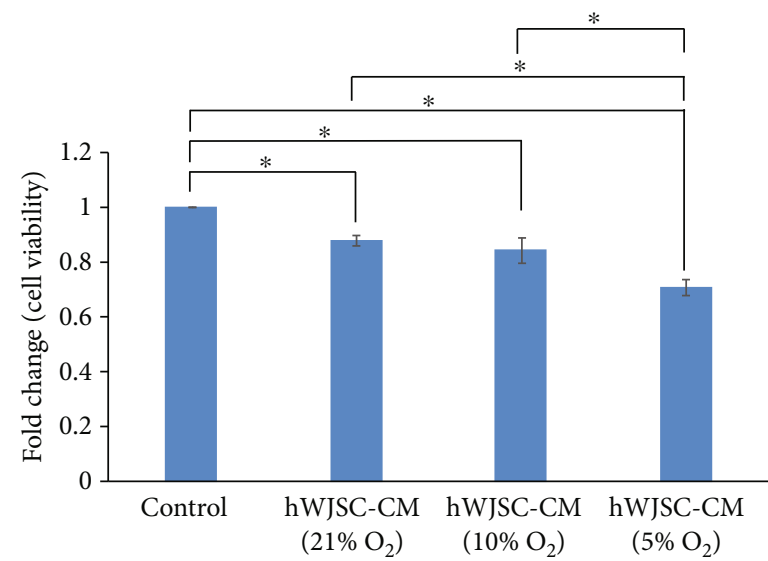

(a)

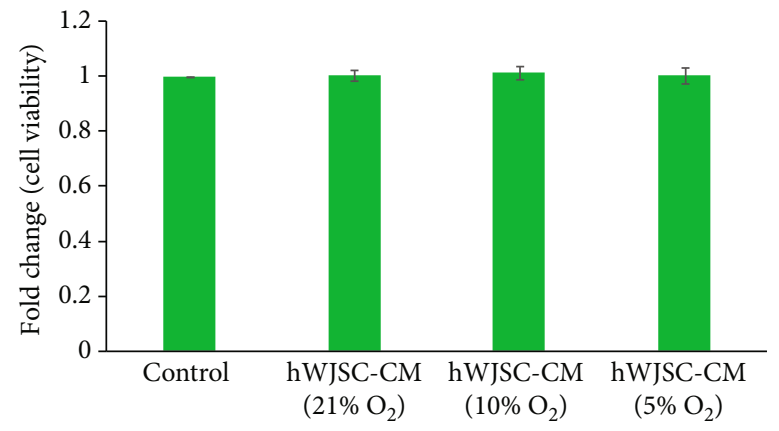

(b)

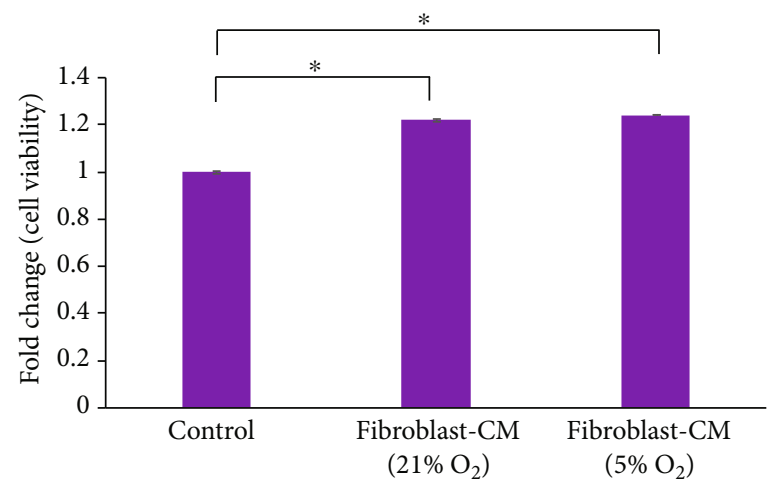

(c)

FIGURE 1: Cell viability (MTS assay) of human lymphoma and control CCD112sk cells exposed to normoxic and hypoxic hWJSC-CM for $48 \mathrm{~h}$. (a) Note significant decreases in cell viability of lymphoma cells after treatment with normoxic or hypoxic hWJSC-CM as compared to controls. Treatment with hWJSC-CM collected under 5\% oxygen conditions showed the greatest decrease of lymphoma cell viability. (b) Note no significant changes to the cell viability of the control cells (skin fibroblasts, CCD112sk) after treatment with hWJSC-CM. (c) Note significant increases in cell viability of lymphoma cells after treatment with normoxic or hypoxic fibroblast-CM as compared to controls. All values are expressed as the mean \pm SEM of 3 biological samples with 3 replicates for each sample. ${ }^{*} p<0.05$.

percentages of early and late apoptotic cells as compared to normoxic hWJSC-CM $\left(21 \% \mathrm{O}_{2}\right)$ and hWJSC-CM $(10 \%$ $\left.\mathrm{O}_{2}\right)$. The values were hWJSC-CM $\left(21 \% \mathrm{O}_{2}\right): 13.0 \pm 0.02 \%$, hWJSC-CM $\left(10 \% \mathrm{O}_{2}\right): 16.6 \pm 0.87 \%$, hWJSC-CM $\left(5 \% \mathrm{O}_{2}\right)$ : $18.9 \pm 0.54 \%$, and control: $8.60 \pm 0.09 \%$. The percentages of late apoptotic cells were hWJSC-CM $\left(21 \% \mathrm{O}_{2}\right): 19.9 \pm 0.31$ $\%$, hWJSC-CM $\left(10 \% \mathrm{O}_{2}\right): 17.6 \pm 0.80 \%$, hWJSC-CM (5\% $\mathrm{O}_{2}$ ): $24.8 \pm 0.22 \%$, and control: $11.7 \pm 0.47 \%$ (Figure $2(\mathrm{a})$ ).

Activated caspase 3, caspase 8, and caspase 9 activities were evaluated using flow cytometry (Figure 2(b)). Significant increases in activated caspase 3 , caspase 8 , and caspase 9 activities were observed in lymphoma cells as compared to controls after $48 \mathrm{~h}$ of treatment with hypoxic and normoxic hWJSC-CM. The hypoxic hWJSC-CM $\left(\begin{array}{lll}5 \% & \mathrm{O}_{2}\end{array}\right)$ induced significantly greater increases in caspase activities as compared to normoxic hWJSC-CM $\left(21 \% \mathrm{O}_{2}\right)$ and hWJSC-CM $\left(10 \% \mathrm{O}_{2}\right)$ (Figure $2(\mathrm{c})$ ). The percentages of lymphoma cells that were positive for activated caspase 3 were hWJSC-CM $\left(21 \% \mathrm{O}_{2}\right): 41.7 \pm 1.00 \%$, hWJSC-CM $(10 \%$ $\left.\mathrm{O}_{2}\right): 41.17 \pm 0.68$, hWJSC-CM $\left(5 \% \mathrm{O}_{2}\right): 57.5 \pm 0.26 \%$, and control: $15.6 \pm 0.40 \%$. The percentages of lymphoma cells that were positive for activated caspase 8 activities were hWJSC-CM $\left(21 \% \mathrm{O}_{2}\right): 32.9 \pm 1.15 \%$, hWJSC-CM (10\% $\left.\mathrm{O}_{2}\right): 31.8 \pm 0.64 \%$, hWJSC-CM $\left(5 \% \mathrm{O}_{2}\right): 46.2 \pm 1.19 \%$, and control: $14.8 \pm 0.56 \%$. The percentages of lymphoma cells that were positive for activated caspase 9 activities were hWJSC-CM $\left(21 \% \mathrm{O}_{2}\right): 27.8 \pm 1.37 \%$, hWJSC-CM $(10 \%$ $\left.\mathrm{O}_{2}\right): 26.0 \pm 1.08 \%$, hWJSC-CM $\left(5 \% \mathrm{O}_{2}\right): 36.3 \pm 0.9 \%$, and control: $13.8 \pm 0.50 \%$.

3.4. Mitochondrial Superoxide, Mitochondrial Membrane Potential, and Lipid Peroxidation of Lymphoma Cells when Treated with Normoxic or Hypoxic hWJSC-CM. Lymphoma cells exposed to both normoxic and hypoxic hWJSC-CM showed significant increases in the number of cells positive for mitochondrial superoxide (MitoSOX) as compared to controls. The hypoxic hWJSC-CM $\left(5 \% \mathrm{O}_{2}\right)$ induced significantly greater increases in the number of cells positive for MitoSOX as compared to normoxic hWJSC-CM $\left(21 \% \mathrm{O}_{2}\right)$ and hypoxic hWJSC-CM $\left(10 \% \mathrm{O}_{2}\right)$. The percentages of cells positive for MitoSOX were hWJSC-CM $\left(21 \% \mathrm{O}_{2}\right): 18.3 \pm$ $0.09 \%$, hWJSC-CM $\left(10 \% \mathrm{O}_{2}\right): 19.6 \pm 0.05 \%$, hWJSC-CM 

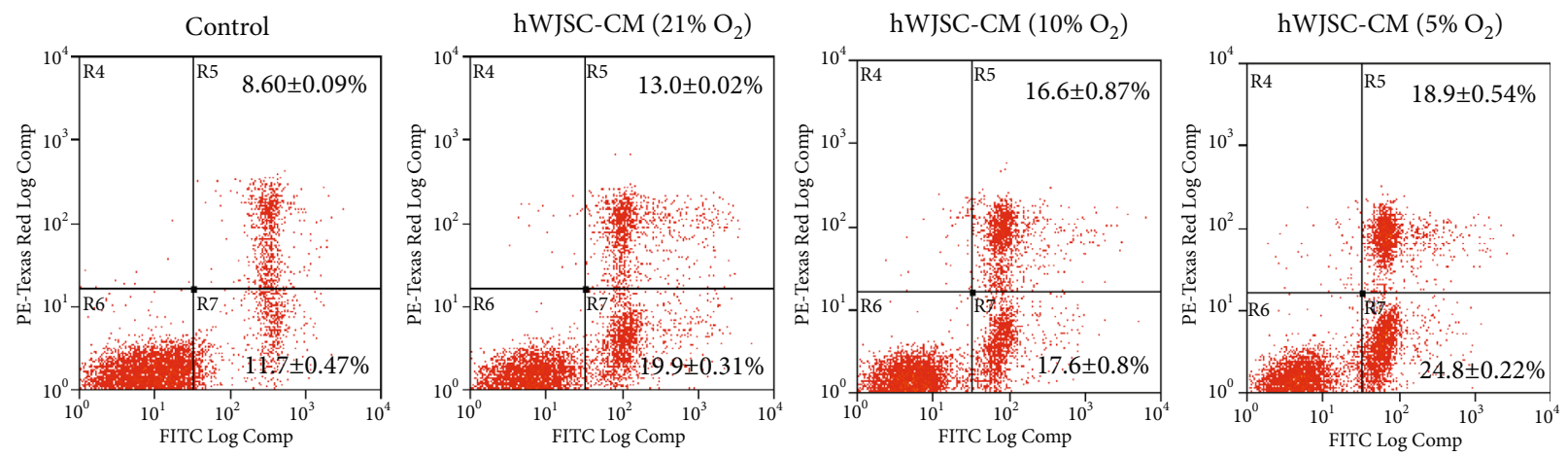

(a)

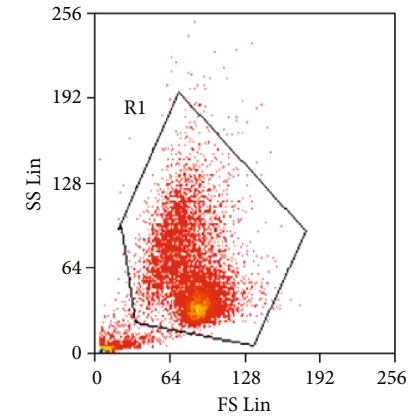

Caspase $3(\%)$

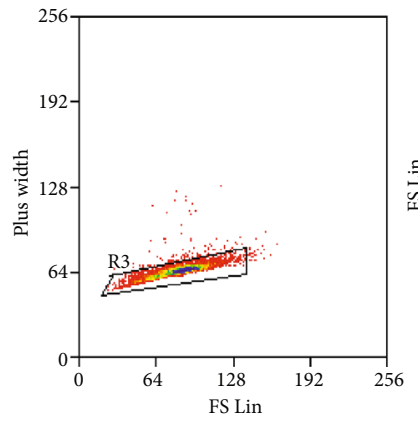

(b)

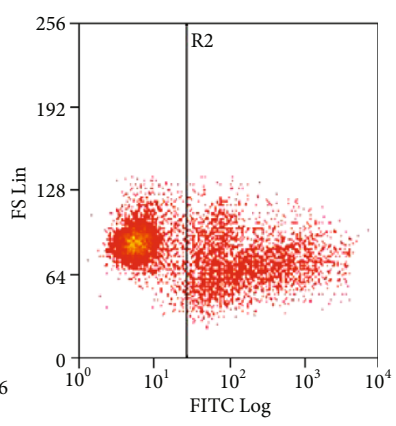

Caspase $8(\%)$
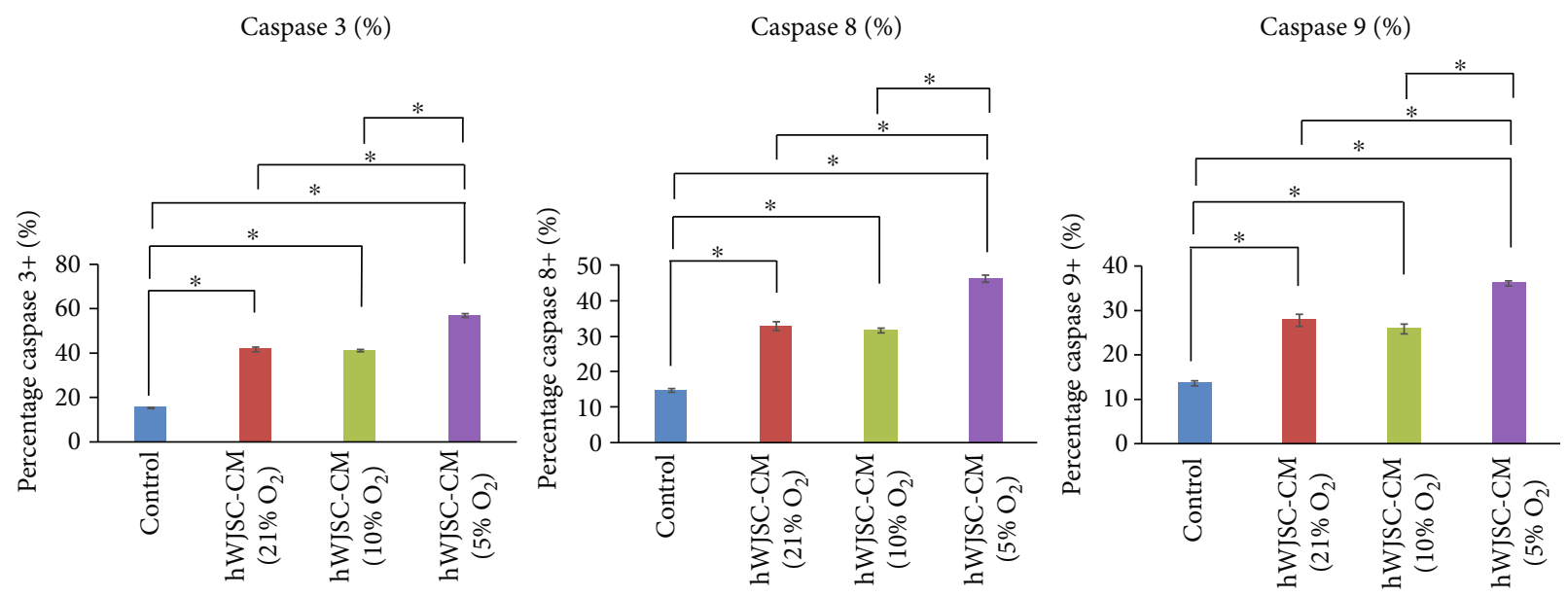

(c)

FIgURE 2: (a) Apoptotic assay (dot plots) of lymphoma cells after treatment with normoxic and hypoxic hWJSC-CM. Note significant increases in percentages of $\mathrm{AV}+\mathrm{PI}-$ and $\mathrm{AV}+\mathrm{PI}+$ of lymphoma cells after treatment with hWJSC-CM as compared to the control. Treatment with hWJSC-CM collected under $5 \%$ oxygen condition showed the highest percentage increase in AV+PI- and AV+PI+ cells. $(b, c)$ Flow cytometry dot plots and percentage caspase activity showed significant increases in caspase 3, caspase 8 , and caspase 9 activities in lymphoma cells after treatment with hWJSC-CM as compared to the control. Treatment with hWJSC-CM collected under 5\% oxygen conditions showed the highest increase in all caspase activities in lymphoma cells. All values are expressed as the mean \pm SEM of 3 biological samples with 3 replicates for each sample. ${ }^{*} p<0.05$.

$\left(5 \% \mathrm{O}_{2}\right): 22.9 \pm 0.06 \%$, and control: $14.4 \pm 0.26 \%$. The mean \pm SEM fluorescence intensities of MitoSOX were hWJSC-CM $\left(21 \% \mathrm{O}_{2}\right): 56.1 \pm 1.41$, hWJSC-CM $\left(10 \% \mathrm{O}_{2}\right)$ : $59.1 \pm 1.00$, hWJSC-CM $\left(5 \% \mathrm{O}_{2}\right): 78.94 \pm 0.95$, and control: $42.5 \pm 0.5$ (Figure 3(a)).

Lymphoma cells exposed to both normoxic and hypoxic hWJSC-CM showed significant decreases in mitochondrial membrane potential (CMXRos) as compared to controls.
The hypoxic hWJSC-CM $\left(\begin{array}{lll}5 \% & \mathrm{O}_{2}\end{array}\right)$ induced significantly greater decreases in mitochondrial membrane potential as compared to normoxic hWJSC-CM $\left(21 \% \mathrm{O}_{2}\right)$ and hypoxic hWJSC-CM $\left(10 \% \mathrm{O}_{2}\right)$. The percentages of cells positive for CMXRos were hWJSC-CM $\left(21 \% \mathrm{O}_{2}\right): 78.3 \pm 0.40 \%$, hWJSC-CM $\left(10 \% \mathrm{O}_{2}\right): 79.3 \pm 0.19 \%$, hWJSC-CM $\left(5 \% \mathrm{O}_{2}\right)$ : $68.2 \pm 0.05 \%$, and control: $80.6 \pm 0.02 \%$. The mean \pm SEM fluorescence intensities of CMXRos were hWJSC-CM $(21 \%$ 

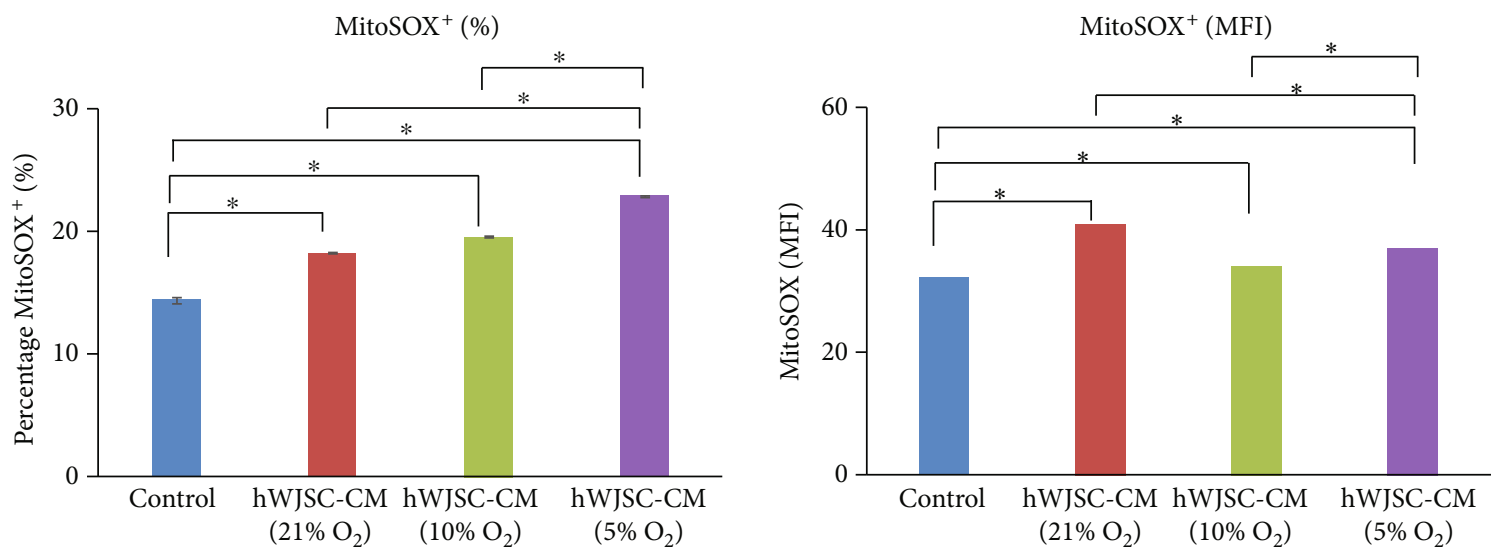

(a)
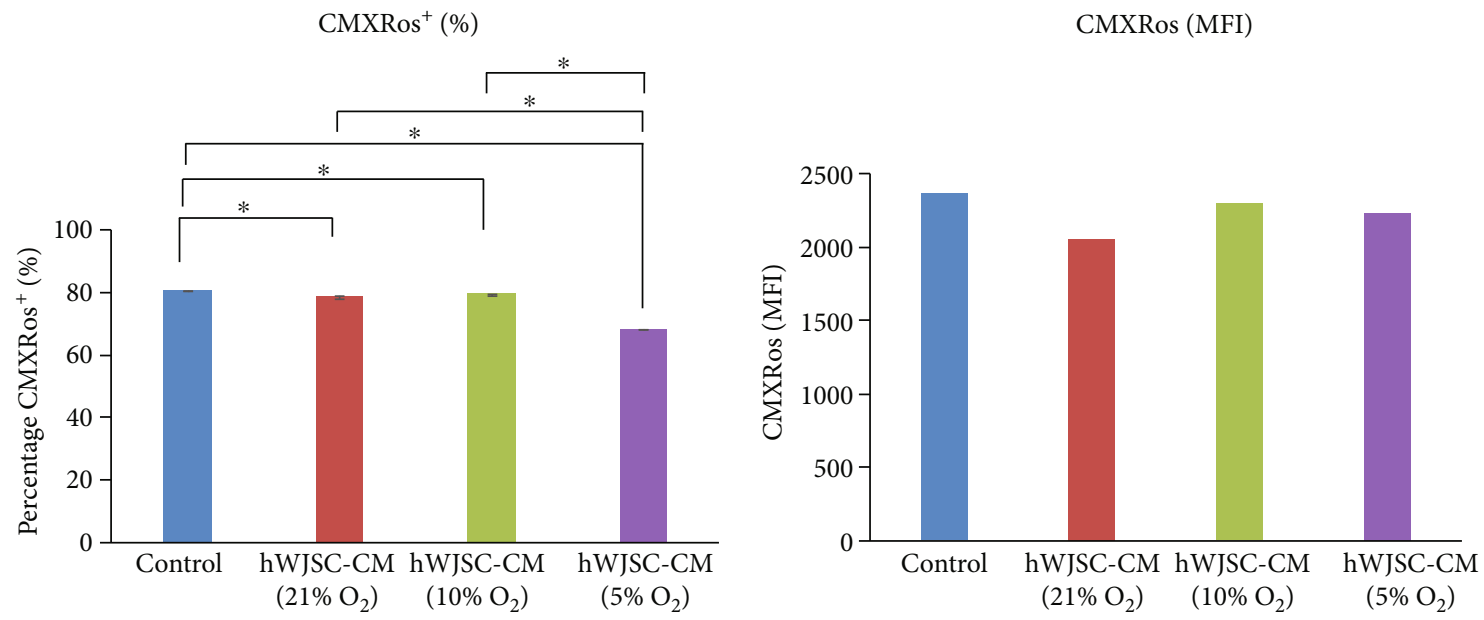

(b)

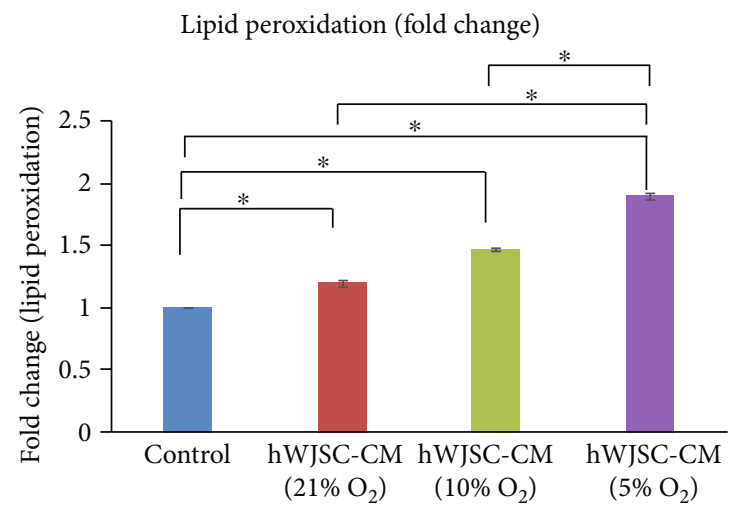

(c)

FIGURE 3: Mitochondria stress assay of lymphoma cells after treatment with normoxic and hypoxic hWJSC-CM. (a) Note significant increases in mitochondria superoxide level of lymphoma cells after treatment with hWJSC-CM as compared to control. Treatment with hWJSC-CM collected under 5\% oxygen conditions showed the highest increase in lymphoma mitochondria superoxide levels. (b) Note significant decreases in mitochondria membrane potential of lymphoma cells after treatment with hWJSC-CM as compared to the control. Treatment with hWJSC-CM collected under 5\% oxygen conditions showed the highest decrease in mitochondria membrane potential. (c) Note significant increases in the lipid peroxidation level of lymphoma cells after treatment with hWJSC-CM as compared to the control. Treatment with hWJSC-CM collected under 5\% oxygen conditions showed the highest increase in lipid peroxidation. All values are expressed as the mean \pm SEM of 3 biological samples with 3 replicates for each sample. ${ }^{*} p<0.05$. 
$\left.\mathrm{O}_{2}\right): 2050.8 \pm 42.2$, hWJSC-CM $\left(10 \% \mathrm{O}_{2}\right): 2290.0 \pm 44.6$, hWJSC-CM $\left(5 \% \mathrm{O}_{2}\right): 2225.5 \pm 27.6$, and control: $2355.3 \pm$ 22.1 (Figure 3(b)).

Lymphoma cells treated with either normoxic or hypoxic hWJSC-CM showed significant fold change increases in lipid peroxidation as compared to controls. The hypoxic hWJSCCM $\left(5 \% \mathrm{O}_{2}\right)$ induced significantly greater fold increases in lipid peroxidation as compared to normoxic hWJSC-CM $\left(21 \% \mathrm{O}_{2}\right)$ and hypoxic hWJSC-CM $\left(10 \% \mathrm{O}_{2}\right)$. The fold changes of lipid peroxidation compared to controls were hWJSC-CM $\left(21 \% \mathrm{O}_{2}\right): 1.2 \pm 0.03$, hWJSC-CM $\left(10 \% \mathrm{O}_{2}\right)$ : $1.47 \pm 0.02$, and hWJSC-CM $\left(\begin{array}{llll}5 \% & \left.\mathrm{O}_{2}\right): & 1.89 \pm 0.03\end{array}\right.$ (Figure 3(c)).

3.5. CRT, Hsp70, Hsp90, CD47, and PD-L1 Expression in Lymphoma Cells Treated with Normoxic or Hypoxic $h W J S C-C M$. There were significant increases in expression of surface bound ecto-CRT, ecto-Hsp70, and ecto-Hsp90 in lymphoma cells treated with normoxic or hypoxic hWJSC$\mathrm{CM}$ as compared to controls. The hypoxic hWJSC-CM (5\% $\mathrm{O}_{2}$ ) induced significantly greater increases in the expression of surface bound CRT, Hsp70, and Hsp90 as compared to normoxic hWJSC-CM $\left(21 \% \mathrm{O}_{2}\right)$ and hypoxic hWJSC-CM $\left(10 \% \mathrm{O}_{2}\right)$. The mean \pm SEM fluorescence intensities of ectoCRT were hWJSC-CM $\left(21 \% \mathrm{O}_{2}\right): 31.0 \pm 0.3$, hWJSC-CM $\left(10 \% \mathrm{O}_{2}\right): 33.8 \pm 1.34$, hWJSC-CM $\left(5 \% \mathrm{O}_{2}\right): 54.8 \pm 1.71$, and control: $20.2 \pm 0.78$ (Figure 4(a)).

The mean \pm SEM fluorescence intensities of ecto-Hsp70 were hWJSC-CM $\left(21 \% \mathrm{O}_{2}\right): 9.01 \pm 0.21$, hWJSC-CM $(10 \%$ $\left.\mathrm{O}_{2}\right): 8.8 \pm 0.70$, hWJSC-CM $\left(5 \% \mathrm{O}_{2}\right): 9.6 \pm 0.21$, and control: $10.5 \pm 0.30$ (Figure 4(b)). The mean \pm SEM fluorescence intensities of ecto-Hsp90 were hWJSC-CM $\left(21 \% \mathrm{O}_{2}\right): 14.2$ \pm 0.21 , hWJSC-CM $\left(10 \% \mathrm{O}_{2}\right): 16.4 \pm 0.46$, hWJSC-CM $\left(5 \% \mathrm{O}_{2}\right): 20.7 \pm 0.3$, and control: $13.2 \pm 0.16$ (Figure 4(c)).

The results showed that there were significant decreases in CD47 expression in lymphoma cells exposed to normoxic or hypoxic hWJSC-CM compared to controls. The hypoxic hWJSC-CM collected at $10 \%$ or $5 \%$ oxygen levels induced significantly greater increases in CD47 expression as compared to normoxic hWJSC-CM $\left(21 \% \mathrm{O}_{2}\right)$. There were no significant differences in CD47 expression between the $10 \%$ and $5 \%$ hypoxic hWJSC-CM-treated lymphoma cells. The mean \pm SEM fluorescence intensities of CD47 were hWJSC-CM $\left(21 \% \mathrm{O}_{2}\right): 98.4 \pm 1.07$, hWJSC-CM $\left(10 \% \mathrm{O}_{2}\right): 89.0 \pm 0.38$, hWJSC-CM $\left(5 \% \mathrm{O}_{2}\right): 90.3 \pm 0.92$, and control: $117.4 \pm 0.11$ (Figure 4(d)).

The results showed that there were significant decreases in PD-L1 expression in lymphoma cells exposed to normoxic or hypoxic hWJSC-CM compared to controls. The hypoxic hWJSC-CM $\left(5 \% \mathrm{O}_{2}\right)$ induced significantly greater decreases in PD-L1 expression as compared to normoxic hWJSC-CM $\left(21 \% \mathrm{O}_{2}\right)$ and hypoxic hWJSC-CM $\left(10 \% \mathrm{O}_{2}\right)$. The mean \pm SEM fluorescence intensities of PD-L1 were hWJSC-CM $\left(21 \% \mathrm{O}_{2}\right): 33.2 \pm 0.48$, hWJSC-CM $\left(10 \% \mathrm{O}_{2}\right): 32.8 \pm 1.10$, hWJSC-CM (5\% $\left.\mathrm{O}_{2}\right): 27.4 \pm 0.61$, and control: $37.5 \pm 1.31$ (Figure 4(e)).

3.6. ATP and HMGB1 Levels in Lymphoma Cells Treated with Normoxic or Hypoxic $h$ WJSC-CM. The results showed that there were no significant differences in extracellular ATP levels in lymphoma cells after treatment with normoxic hWJSC-CM $\left(21 \% \mathrm{O}_{2}\right)$ or hypoxic hWJSC-CM $\left(10 \% \mathrm{O}_{2}\right.$ and $5 \% \mathrm{O}_{2}$ ) compared to controls. The mean optical density readings measured at $570 \mathrm{~nm}$ for ATP levels were hWJSC-CM $\left(21 \% \mathrm{O}_{2}\right): 0.08 \pm 0.001$, hWJSC-CM $\left(10 \% \mathrm{O}_{2}\right): 0.07 \pm 0.004$ , hWJSC-CM (5\% $\left.\mathrm{O}_{2}\right): 0.08 \pm 0.001$, and control: $0.07 \pm$ 0.004 (Figure 5(a)).

The results showed that there were significant increases in extracellular HMGB1 levels in lymphoma cells after exposure to normoxic hWJSC-CM $\left(21 \% \mathrm{O}_{2}\right)$ or hypoxic hWJSC$\mathrm{CM}\left(5 \% \mathrm{O}_{2}\right)$ compared to controls. However, there were no significant differences in extracellular HMGB1 levels observed between normoxic hWJSC-CM $\left(21 \% \mathrm{O}_{2}\right)$ and hypoxic hWJSC-CM $\left(5 \% \mathrm{O}_{2}\right)$. The mean optical density readings measured at $450 \mathrm{~nm}$ for HMGB1 levels were hWJSC-CM $\left(21 \% \mathrm{O}_{2}\right): 0.28 \pm 0.002$, hWJSC-CM $\left(10 \% \mathrm{O}_{2}\right): 0.24 \pm 0.02$, hWJSC-CM ( $\left.5 \% \mathrm{O}_{2}\right): 0.27 \pm 0.005$, and control: $0.22 \pm$ 0.003 (Figure $5(\mathrm{~b})$ ).

\section{Discussion}

The human umbilical cord provides for the transmission of nutrition from mother to fetus. The gelatinous nature of intervascular Wharton's jelly helps to prevent the strangulation of the umbilical blood vessels cutting off the nutritional supply to the fetus. Additionally, the unique tumoricidal properties of the stem cells lying within Wharton's jelly (hWJSCs) help to engulf and destroy any cancer cells that migrate from mother to fetus thus explaining why cancers of the fetus are extremely low in mothers who are pregnant and ill with various carcinomas [28-33].

The hWJSCs possess all the characteristics of bona fide MSCs [34], but unlike bone marrow MSCs, they do not form into cancer-associated fibroblasts in tumor environments [35-37]. hWJSCs also do not induce tumorigenesis and toxicity in laboratory animal models, cynomolgus monkeys, and in clinical trials, which suggests that they are safe when used in clinical settings $[5,38]$. In almost all of these preclinical and clinical trials studied thus far, hWJSCs were maintained and cultured in normoxic conditions instead of their physiological oxygen tension levels of $5 \%$ and below. It is therefore important to evaluate whether the positive tumoricidal results obtained thus far with hWJSCs grown under normoxic conditions can be maintained or are superior when they are grown and applied under physiological hypoxic conditions.

MSCs in general including hWJSCs pass through two different phases of oxygen conditions from their in vivo collection, derivation in vitro and back to in vivo transplantation. Conventionally, in most culture conditions, the stem cells are usually exposed to higher oxygen concentrations during their in vitro manipulation compared to much lower concentrations when they are in vivo. Oxygen is vital in maintaining stemness, expanded growth, and differentiation of stem cells via regulation of hypoxia-inducible factor-1- (HIF1-a-) related gene expression. The culture of stem cells at a higher oxygen concentration as compared to the lower concentration in their natural niches induces environmental stress, 


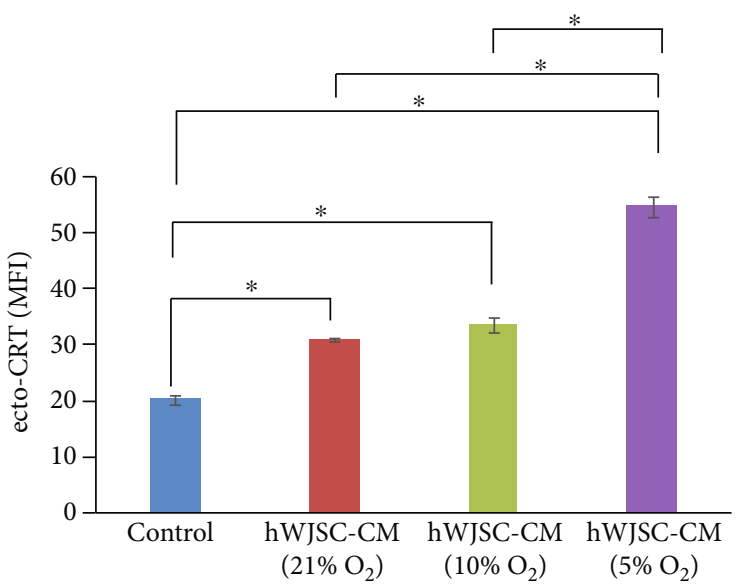

(a)

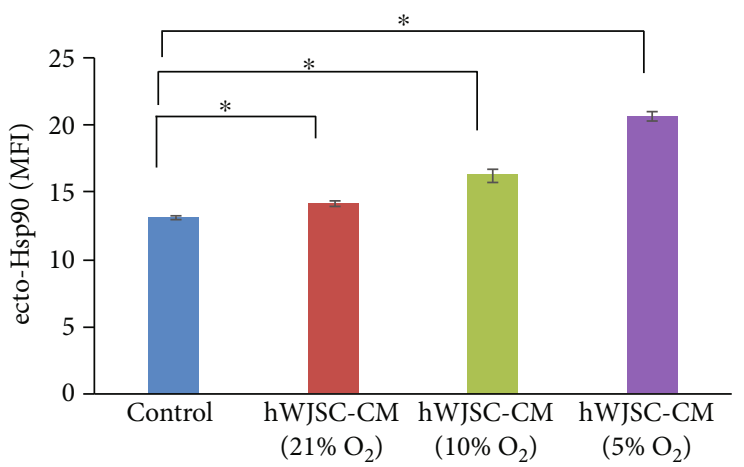

(c)

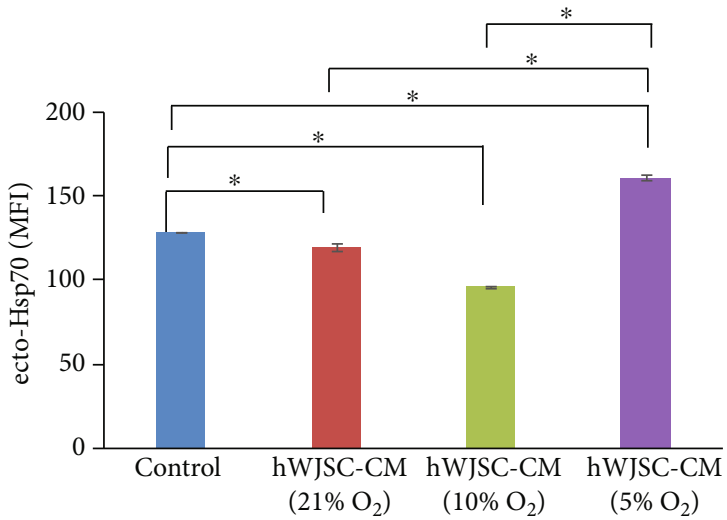

(b)

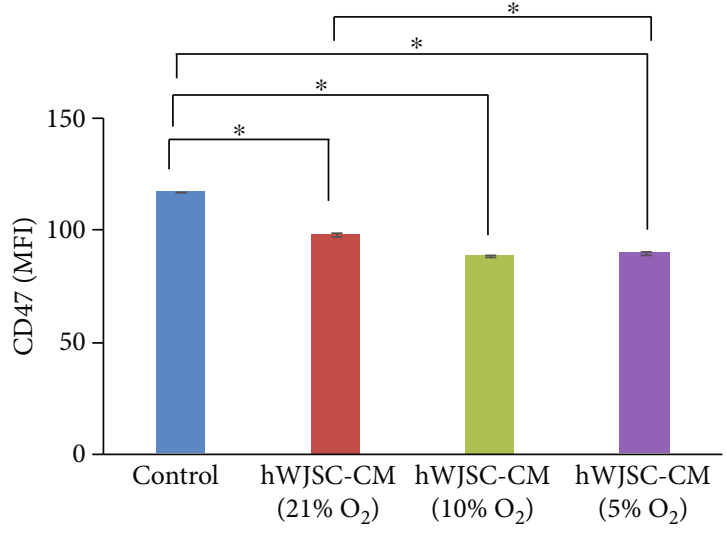

(d)

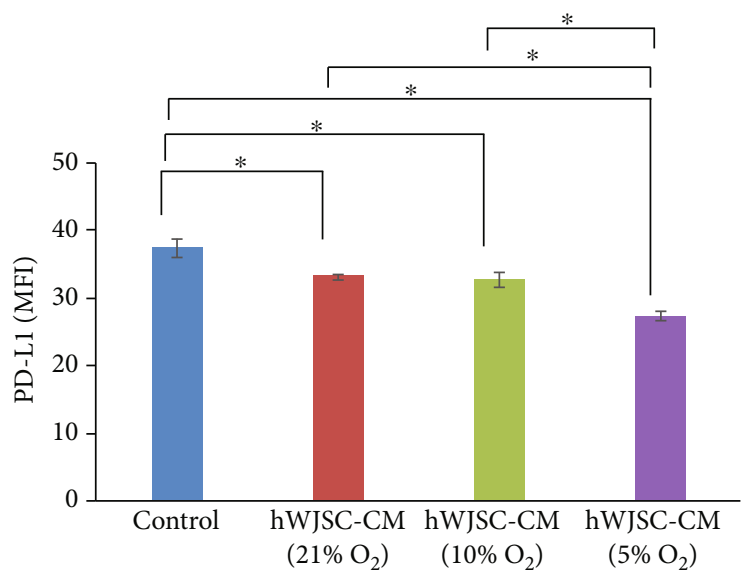

(e)

FIgURE 4: Immunogenic cell death analysis of lymphoma cells after treatment with normoxic and hypoxic hWJSC-CM. (a-c) Note significantly higher ecto-CRT, ecto-Hsp70, and ecto-Hsp90 expression after treatment with hypoxic 5\% oxygen hWJSC-CM as compared to the control. (d, e) Note significantly lower CD47 and PD-L1 expressions in lymphoma cells after treatment with hWJSC-CM as compared to the control. Treatment with hWJSC-CM collected under 5\% oxygen conditions showed the highest and lowest "find me/eat me" and "don't eat me" signals in lymphoma cells, respectively. All values are expressed as the mean \pm SEM of 3 biological samples with 3 replicates for each sample. ${ }^{*} p<0.05$.

early cell ageing, slower growth, and chromatin damage which leads to poor engraftment and suboptimal clinical results [39].
Culturing of hWJSCs at higher oxygen concentrations may have similar detrimental effects too. We observed in the present study that hWJSCs cultured under hypoxic 


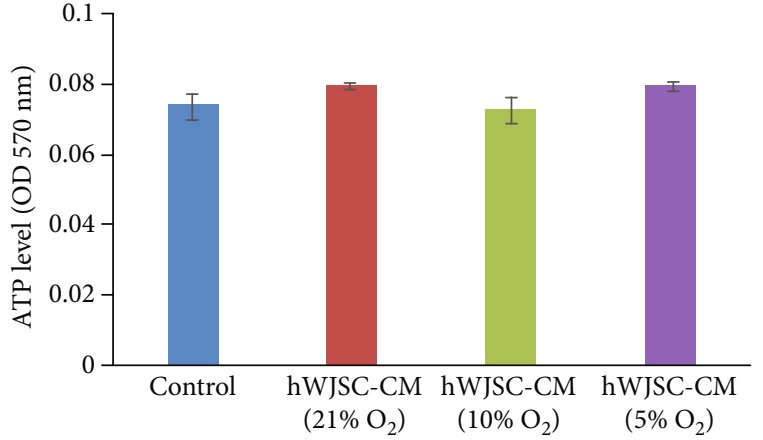

(a)

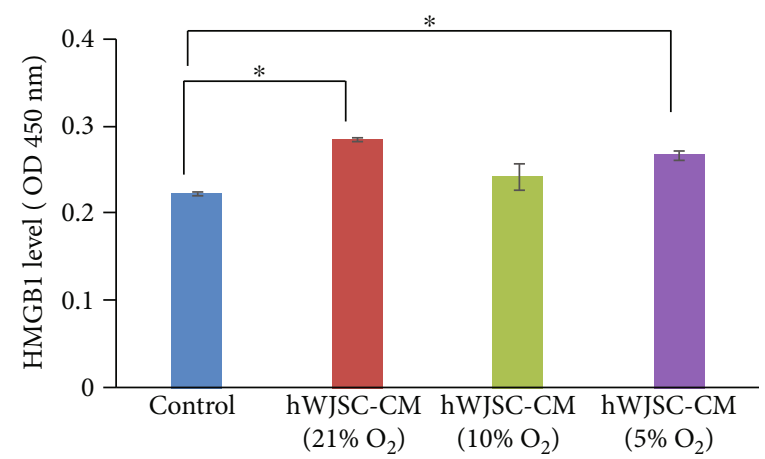

(b)

Figure 5: Secreted ATP and HMGB1 levels of lymphoma cells after treatment with normoxic and hypoxic hWJSC-CM. (a) Note no significant difference in extracellular ATP levels in lymphoma cells after treatment with normoxic and hypoxic hWJSC-CM as compared to the control. (b) Note significantly higher extracellular HMGB1 levels in lymphoma cells after treatment with normoxic and hypoxic 5\% oxygen hWJSC-CM as compared to the control. All values are expressed as the mean \pm SEM of 3 biological samples with 3 replicates for each sample. ${ }^{*} p<0.05$.

conditions showed similar cell morphologies and could undergo trilineage differentiation into the adipocytes, osteocytes, and chondrocytes like that of normoxic hWJSC-CM (Supplemental Figure 1). Also, hWJSCs grown in hypoxic conditions $(10 \%$ or $5 \%)$ remained positive for the MSC markers CD105, CD90, CD73, CD146, and CD108, and the expression levels of these markers were significantly greater than those for normoxic hWJSCs. Our results support those of other groups where there was no change in the phenotype markers when cultured in hypoxic or normoxic conditions [40, 41].

Although our results showed that normoxic and hypoxic hWJSC-CM induced significant reduction in lymphoma cell viability and showed increased numbers of apoptotic cells, the exposure to hypoxic hWJSC-CM (5\% $\mathrm{O}_{2}$ ) showed the greatest cell viability reduction and apoptotic cell numbers. These tumoricidal effects are consistent with previous published studies where hypoxic WJSC-CM exhibited greater attenuation of cell growth of cervical, liver, prostate, and ovarian cancers as compared to normoxic WJSC-CM [40, 41]. We also observed that treatment of lymphoma cells with hypoxic hWJSC-CM induced the highest oxidative stress in the mitochondria and with the highest levels of lipid peroxidation as compared to normoxic hWJSC-CM. The presentation of "find-me/eat-me"danger-associated molecular pattern (DAMP) molecules and the reduced expression of the "don't eat me" signals (PD-L1 and CD47) in the dying cancer cells trigger an immune response by the host and achieve complete remission $[19,20]$. There is growing evidence that anticancer agents that could stimulate tumor-specific responses via immunogenic cell death improved the efficacy and longterm therapeutic success [42]. Likewise, strategies to block CD47 and PD-L1 expressions on cancer cells are of immense interest as they enhance the antitumor response $[19,43]$. Our study showed that treatment with hypoxic hWJSC-CM at $5 \%$ oxygen tensions had the greatest upregulation of the DAMPs such as ecto-CRT, ecto-Hsp70, Hsp90, and HMGB1 and downregulation of CD47 and PD-L1.
We hypothesize that culturing hWJSCs and collection of hWJSC-CM in normoxic and 10\% hypoxic conditions may not produce differences in the various types of factors (secretome) in the hWJSC-CM. As such, we did not see significant differences in the anticancer effect between normoxic hWJSC-CM and 10\% oxygen hWJSC-CM. The differences in the anticancer effect were observed when we cultured hWJSCs and collected hWJSC-CM in 5\% hypoxic conditions. This coincides with the literature that suggests that the physiological oxygen tension in the umbilical cord is about $5 \%$ (between $2 \%$ and $8 \%$ ).

The results suggest that using $5 \%$ hypoxic hWJSC-CM will elicit a greater antitumor immunity response as compared to hWJSC-CM prepared under the typical normoxic culture conditions.

The increased tumoricidal effect of hWJSC-CM prepared under hypoxic conditions may be due to increased expression and secretion of anticancer molecules or microvesicles/exosomes as compared to hWJSC-CM prepared under normoxic conditions. Expression of HIF1-a was also previously shown to be upregulated in hypoxic conditions and in turn regulate many downstream process stemness and differentiation of stem cells [39]. We have previously reported that hWJSC-CM contains significantly higher levels of interleukins and adhesion molecules like IL-1a, IL-6, IL-8, HGF, SCF, and MCP-1 that regulate cancer cell death and modulate the immune system [17]. We have also reported that hWJSCs highly express miRNA-146a and miRNA-126 that target the innate and adaptive immune system [44]. The expression of miRNA-146a and miRNA-126 has been reported to activate the $\mathrm{T}$ cell immunity or prevent the growth of various cancer cells [45-53]. Further studies aimed at comparatively evaluating the hypoxic and normoxic hWJSC-CM secretome and HIF1-a pathways will give further insights on the various molecules involved in the increased tumoricidal and immunogenic cell death observed with hypoxic hWJSC-CM which can eventually be characterized and tested for their tumoricidal effects singly or in combinations. 
Though this study evaluated the differences in tumoricidal effects of hWJSCs cultured under different oxygen concentrations, there is a lack of information on the exposure of hWJSCs to atmospheric oxygen concentrations during the isolation or derivation process. It has been demonstrated that brief exposure to ambient oxygen concentrations during the collection and isolation has rapid and irreversible changes to the metabolism in cord blood hematopoietic stem cells or progenitor cells (HSC/HPC) [54]. The cells undergo extra physiologic oxygen shock/stress (EPHOSS) where they produce ambient air-induced mitochondrial ROS which initiate the opening of the mitochondrial permeability transition pore [55]. Collection, processing, and culturing of HSC/HPCs under hypoxic conditions enhanced the collection and proliferation of long-term repopulating HSCs. However, the collection and processing at low oxygen tensions are a huge logistical problem that is both cumbersome and expensive. The addition of cyclosporin A, an FDAapproved drug, during the collection and processing of cord blood has been shown to mitigate the effect of EPHOSS by mimicking the effects of low oxygen concentration [54]. As most of the EPHOSS effect studies were on HSCs/HPCs [54], there is limited information on the EPHOSS on MSCs and hWJSCs. More studies are needed to evaluate the metabolism of hWJSCs affected by EPHOSS and whether the addition of cyclosporin A into the processes could generate hWJSCs with higher therapeutic potential.

\section{Conclusion}

Our results suggest that hypoxic hWJSC-CM induces a greater tumoricidal response via the presentation of DAMPs to initiate antitumor immunity. Hypoxic hWJSC-CM could also suppress the defense molecule expression on lymphoma cells that could make the lymphoma cells more prone to an immune response by the host in order to achieve complete remission. Although both normoxic and hypoxic hWJSC$\mathrm{CM}$ hold tremendous promise as novel agents against malignant tumors, hypoxic hWJSC-CM should be the agent of choice because of its greater tumoricidal effects.

\section{Data Availability}

The data used to support the findings of this study are included within the article.

\section{Conflicts of Interest}

All authors declare that they have no conflicts of interest.

\section{Acknowledgments}

The authors thank Ms. Jasmine Ge for her assistance. This research is supported by the Singapore National University Health System (NUHS) Aspiration Fund (New Idea) grant (R-174-000-155-720) and the NUHS Department of Obstetrics and Gynecology Pitch For Funds Round (PFFR) grant (2015).

\section{Supplementary Materials}

Morphology and trilineage differentiation of hWJSCs cultured under normoxic $\left(21 \% \mathrm{O}_{2}\right)$ and hypoxic $(10 \%$ or $5 \%)$ conditions. (A) hWJSCs cultured in normoxic and hypoxic conditions were plastic adherent and showed no distinct morphological difference between them. (B) Both normoxic and hypoxic hWJSCs differentiated into adipocytes after 21 days in adipogenesis differentiation medium. The normoxic and hypoxic hWJSCs exhibited lipid droplets with Oil Red O staining. (C) Both normoxic and hypoxic hWJSCs differentiated into osteocytes after 21 days in osteogenic differentiation medium. The normoxic and hypoxic hWJSCs exhibited calcium deposition with Von Kossa staining. (D) Both normoxic and hypoxic hWJSCs differentiated into chondrocytes after 21 days in chondrogenic differentiation medium. The normoxic and hypoxic hWJSCs exhibited glycosaminoglycans with Alcian blue staining. (Supplementary Materials)

\section{References}

[1] C. Y. Fong, M. Richards, N. Manasi, A. Biswas, and A. Bongso, "Comparative growth behaviour and characterization of stem cells from human Wharton's jelly," Reproductive Biomedicine Online, vol. 15, no. 6, pp. 708-718, 2007.

[2] C. Y. Fong, A. Subramanian, A. Biswas et al., "Derivation efficiency, cell proliferation, freeze-thaw survival, stem-cell properties and differentiation of human Wharton's jelly stem cells," Reproductive Biomedicine Online, vol. 21, no. 3, pp. 391-401, 2010.

[3] X. Y. Wang, Y. Lan, W. Y. He et al., "Identification of mesenchymal stem cells in aorta-gonad-mesonephros and yolk sac of human embryos," Blood, vol. 111, no. 4, pp. 24362443, 2008.

[4] A. Bongso and C. Y. Fong, "The therapeutic potential, challenges and future clinical directions of stem cells from the Wharton's jelly of the human umbilical cord," Stem Cell Reviews, vol. 9, no. 2, pp. 226-240, 2013.

[5] I. Kalaszczynska and K. Ferdyn, "Wharton's Jelly derived mesenchymal stem cells: future of regenerative medicine? Recent findings and clinical significance," BioMed Research International, vol. 2015, Article ID 430847, 11 pages, 2015.

[6] R. Ayuzawa, C. Doi, R. S. Rachakatla et al., "Naïve human umbilical cord matrix derived stem cells significantly attenuate growth of human breast cancer cells in vitro and in vivo," Cancer Letters, vol. 280, no. 1, pp. 31-37, 2009.

[7] K. C. Chao, H. T. Yang, and M. W. Chen, "Human umbilical cord mesenchymal stem cells suppress breast cancer tumourigenesis through direct cell-cell contact and internalization," Journal of Cellular and Molecular Medicine, vol. 16, no. 8, pp. 1803-1815, 2012.

[8] C. Ganta, D. Chiyo, R. Ayuzawa et al., "Rat umbilical cord stem cells completely abolish rat mammary carcinomas with no evidence of metastasis or recurrence 100 days post-tumor cell inoculation," Cancer Research, vol. 69, no. 5, pp. 1815-1820, 2009.

[9] K. Gauthaman, F. C. Yee, S. Cheyyatraivendran, A. Biswas, M. Choolani, and A. Bongso, "Human umbilical cord Wharton's jelly stem cell (hWJSC) extracts inhibit cancer cell growth in vitro," Journal of Cellular Biochemistry, vol. 113, no. 6, pp. 2027-2039, 2012. 
[10] K. Gauthaman, C. Y. Fong, S. Arularasu et al., "Human Wharton's jelly stem cell conditioned medium and cell-free lysate inhibit human osteosarcoma and mammary carcinoma cell growth in vitro and in xenograft mice," Journal of Cellular Biochemistry, vol. 114, no. 2, pp. 366-377, 2013.

[11] A. Kawabata, N. Ohta, G. Seiler et al., "Naïve rat umbilical cord matrix stem cells significantly attenuate mammary tumor growth through modulation of endogenous immune responses," Cytotherapy, vol. 15, no. 5, pp. 586-597, 2013.

[12] Y. Ma, X. Hao, S. Zhang, and J. Zhang, "The in vitro and in vivo effects of human umbilical cord mesenchymal stem cells on the growth of breast cancer cells," Breast Cancer Research and Treatment, vol. 133, no. 2, pp. 473-485, 2012.

[13] T. Matsuzuka, R. S. Rachakatla, C. Doi et al., "Human umbilical cord matrix-derived stem cells expressing interferon-beta gene significantly attenuate bronchioloalveolar carcinoma xenografts in SCID mice," Lung Cancer, vol. 70, no. 1, pp. 28-36, 2010.

[14] D. K. Maurya, C. Doi, A. Kawabata et al., "Therapy with unengineered naïve rat umbilical cord matrix stem cells markedly inhibits growth of murine lung adenocarcinoma," BMC Cancer, vol. 10, no. 1, p. 590, 2010.

[15] S. Wu, G. Q. Ju, T. Du, Y. J. Zhu, and G. H. Liu, "Microvesicles derived from human umbilical cord Wharton's jelly mesenchymal stem cells attenuate bladder tumor cell growth in vitro and in vivo," PLoS One, vol. 8, no. 4, article e61366, 2013.

[16] J. Liu, G. Han, H. Liu, and C. Qin, "Suppression of cholangiocarcinoma cell growth by human umbilical cord mesenchymal stem cells: a possible role of Wnt and Akt signaling," PLoS One, vol. 8, no. 4, article e62844, 2013.

[17] C. Y. Fong, K. Gauthaman, S. Cheyyatraivendran, H. D. Lin, A. Biswas, and A. Bongso, "Human umbilical cord Wharton's jelly stem cells and its conditioned medium support hematopoietic stem cell expansion ex vivo," Journal of Cellular Biochemistry, vol. 113, no. 2, pp. 658-668, 2012.

[18] D. H. Lin, A. Biswas, M. Choolani, C. Y. Fong, and A. Bongso, "Induction of immunogenic cell death in lymphoma cells by Wharton's jelly Mesenchymal stem Cell conditioned medium," Stem Cell Reviews and Reports, vol. 13, no. 6, pp. 801-816, 2017.

[19] L. Bracci, G. Schiavoni, A. Sistigu, and F. Belardelli, "Immunebased mechanisms of cytotoxic chemotherapy: implications for the design of novel and rationale-based combined treatments against cancer," Cell Death and Differentiation, vol. 21, no. 1, pp. 15-25, 2014.

[20] S. C. Casey, L. Tong, Y. Li et al., "MYC regulates the antitumor immune response through CD47 and PD-L1," Science, vol. 352, no. 6282, pp. 227-231, 2016.

[21] B. Fischer and B. D. Bavister, "Oxygen tension in the oviduct and uterus of rhesus monkeys, hamsters and rabbits," Journal of Reproduction and Fertility, vol. 99, no. 2, pp. 673-679, 1993.

[22] T. Ma, W. L. Grayson, M. Frohlich, and G. Vunjak-Novakovic, "Hypoxia and stem cell-based engineering of mesenchymal tissues," Biotechnology Progress, vol. 25, no. 1, pp. 32-42, 2009.

[23] J. Beegle, K. Lakatos, S. Kalomoiris et al., "Hypoxic preconditioning of mesenchymal stromal cells induces metabolic changes, enhances survival, and promotes cell retention in vivo," Stem Cells, vol. 33, no. 6, pp. 1818-1828, 2015.

[24] S. W. Song, K. E. Kim, J. W. Choi et al., "Proteomic analysis and identification of paracrine factors in mesenchymal stem cell-conditioned media under hypoxia," Cellular Physiology and Biochemistry, vol. 40, no. 1-2, pp. 400-410, 2016.

[25] S. C. Lee, H. J. Jeong, S. K. Lee, and S. J. Kim, "Hypoxic conditioned medium from human adipose-derived stem cells promotes mouse liver regeneration through JAK/STAT3 signaling," Stem Cells Translational Medicine, vol. 5, no. 6, pp. 816-825, 2016.

[26] Y. F. Huang, M. J. Chen, M. H. Wu, and S. C. Hung, “The use of hypoxic cultured mesenchymal stem cell for oncolytic virus therapy," Cancer Gene Therapy, vol. 20, no. 5, pp. 308-316, 2013.

[27] Y. Feng, M. Zhu, S. Dangelmajer et al., "Hypoxia-cultured human adipose-derived mesenchymal stem cells are nononcogenic and have enhanced viability, motility, and tropism to brain cancer," Cell Death \& Disease, vol. 5, no. 12, article e1567, 2014.

[28] A. Alexander, W. E. Samlowski, D. Grossman et al., "Metastatic melanoma in pregnancy: risk of transplacental metastases in the infant," Journal of Clinical Oncology, vol. 21, no. 11, pp. 2179-2186, 2003.

[29] G. A. Dildy III, K. J. Moise Jr., R. J. Carpenter Jr., and T. Klima, "Maternal malignancy metastatic to the products of conception: a review," Obstetrical \& Gynecological Survey, vol. 44, no. 7, pp. 535-540, 1989.

[30] C. Jackisch, F. Louwen, A. Schwenkhagen et al., "Lung cancer during pregnancy involving the products of conception and a review of the literature," Archives of Gynecology and Obstetrics, vol. 268, no. 2, pp. 69-77, 2003.

[31] J. Liu and L. Guo, "Intraplacental choriocarcinoma in a term placenta with both maternal and infantile metastases: a case report and review of the literature," Gynecologic Oncology, vol. 103, no. 3, pp. 1147-1151, 2006.

[32] J. F. Potter and M. Schoeneman, "Metastasis of maternal cancer to the placenta and fetus," Cancer, vol. 25, no. 2, pp. 380388, 1970.

[33] H. T. Yang and K. C. Chao, "Foetal defence against cancer: a hypothesis," Journal of Cellular and Molecular Medicine, vol. 17, pp. 1096-1098, 2013.

[34] M. Dominici, K. le Blanc, I. Mueller et al., "Minimal criteria for defining multipotent mesenchymal stromal cells. The International Society for Cellular Therapy position statement," Cytotherapy, vol. 8, no. 4, pp. 315-317, 2006.

[35] A. Subramanian, G. Shu-Uin, N. Kae-Siang et al., "Human umbilical cord Wharton's jelly mesenchymal stem cells do not transform to tumor-associated fibroblasts in the presence of breast and ovarian cancer cells unlike bone marrow mesenchymal stem cells," Journal of Cellular Biochemistry, vol. 113, no. 6, pp. 1886-1895, 2012.

[36] K. Gauthaman, C. Y. Fong, C. A. Suganya et al., "Extra-embryonic human Wharton's jelly stem cells do not induce tumorigenesis, unlike human embryonic stem cells," Reproductive Biomedicine Online, vol. 24, no. 2, pp. 235-246, 2012.

[37] R. N. Bárcia, J. M. Santos, M. Filipe et al., "What makes umbilical cord tissue-derived mesenchymal stromal cells superior immunomodulators when compared to bone marrow derived mesenchymal stromal cells?," Stem Cells International, vol. 2015, Article ID 583984, 14 pages, 2015.

[38] Y. Wang, Z. B. Han, J. Ma et al., "A toxicity study of multipleadministration human umbilical cord mesenchymal stem cells in cynomolgus monkeys," Stem Cells and Development, vol. 21, no. 9, pp. 1401-1408, 2012. 
[39] N. Haque, M. T. Rahman, N. H. Abu Kasim, and A. M. Alabsi, "Hypoxic culture conditions as a solution for mesenchymal stem cell based regenerative therapy," The Scientific World Journal, vol. 2013, Article ID 632972, 12 pages, 2013.

[40] W. Widowati, L. Wijaya, H. Murti et al., "Conditioned medium from normoxia (WJMSCs-norCM) and hypoxiatreated WJMSCs (WJMSCs-hypoCM) in inhibiting cancer cell proliferation," Biomarkers and Genomic Medicine, vol. 7, no. 1, pp. 8-17, 2015.

[41] K.-H. Han, A.-K. Kim, G.-J. Jeong, H. R. Jeon, S. H. Bhang, and D. I. Kim, "Enhanced anti-cancer effects of conditioned medium from hypoxic human Umbilical cord-derived mesenchymal stem cells," International Journal of Stem Cells, vol. 12, no. 2, pp. 291-303, 2019.

[42] H. Inoue and K. Tani, "Multimodal immunogenic cancer cell death as a consequence of anticancer cytotoxic treatments," Cell Death and Differentiation, vol. 21, no. 1, pp. 39-49, 2014.

[43] H. Miyoshi, J. Kiyasu, T. Kato et al., "PD-L1 expression on neoplastic or stromal cells is respectively a poor or good prognostic factor for adult T-cell leukemia/lymphoma," Blood, vol. 128, no. 10, pp. 1374-1381, 2016.

[44] C. Y. Fong, K. Tam, S. Cheyyatraivendran et al., "Human Wharton's jelly stem cells and its conditioned medium enhance healing of excisional and diabetic wounds," Journal of Cellular Biochemistry, vol. 115, no. 2, pp. 290-302, 2014.

[45] L. Paladini, L. Fabris, G. Bottai, C. Raschioni, G. A. Calin, and L. Santarpia, "Targeting microRNAs as key modulators of tumor immune response," Journal of Experimental \& Clinical Cancer Research, vol. 35, no. 1, p. 103, 2016.

[46] A. Mehta and D. Baltimore, "MicroRNAs as regulatory elements in immune system logic," Nature Reviews Immunology, vol. 16, no. 5, pp. 279-294, 2016.

[47] J. Mei, R. Bachoo, and C. L. Zhang, "MicroRNA-146a inhibits glioma development by targeting Notch1," Molecular and Cellular Biology, vol. 31, no. 17, pp. 3584-3592, 2011.

[48] B. Xu, N. Wang, X. Wang et al., "miR-146a suppresses tumor growth and progression by targeting EGFR pathway and in a p-ERK-dependent manner in castration-resistant prostate cancer," Prostate, vol. 72, no. 11, pp. 1171-1178, 2012.

[49] G. Chen, I. A. Umelo, S. Lv et al., "miR-146a inhibits cell growth, cell migration and induces apoptosis in non-small cell lung cancer cells," PLoS One, vol. 8, no. 3, article e60317, 2013.

[50] Q. Yu, S. L. Liu, H. Wang, G. Shi, P. Yang, and X. L. Chen, "miR-126 suppresses the proliferation of cervical cancer cells and alters cell sensitivity to the chemotherapeutic drug bleomycin," Asian Pacific Journal of Cancer Prevention, vol. 14, no. 11, pp. 6569-6572, 2013.

[51] E. Miko, Z. Margitai, Z. Czimmerer et al., "miR-126 inhibits proliferation of small cell lung cancer cells by targeting SLC7A5," FEBS Letters, vol. 585, no. 8, pp. 1191-1196, 2011.

[52] C. Du, Z. Lv, L. Cao et al., "miR-126-3p suppresses tumor metastasis and angiogenesis of hepatocellular carcinoma by targeting LRP6 and PIK3R2," Journal of Transational Medicine, vol. 12, no. 1, p. 259, 2014.

[53] Z. Li, N. Li, M. Wu, X. Li, Z. Luo, and X. Wang, "Expression of miR-126 suppresses migration and invasion of colon cancer cells by targeting CXCR4," Molecular and Cellular Biochemistry, vol. 381, no. 1-2, pp. 233-242, 2013.
[54] H. E. Broxmeyer, H. A. O'Leary, X. Huang, and C. Mantel, "The importance of hypoxia and extra physiologic oxygen shock/stress for collection and processing of stem and progenitor cells to understand true physiology/pathology of these cells ex vivo," Current Opinion in Hematology, vol. 22, no. 4, pp. 273-278, 2015.

[55] C. R. Mantel, H. A. O'Leary, B. R. Chitteti et al., "Enhancing hematopoietic stem cell transplantation efficacy by mitigating oxygen shock," Cell, vol. 161, no. 7, pp. 1553-1565, 2015. 